\title{
The effect of electronic energy loss on the dynamics of thermal spikes in $\mathrm{Cu}$
}

\author{
S. Prönnecke, A. Caro, and M. Victoria \\ Paul Scherrer Institute, 5232 Villigen, Switzerland \\ T. Diaz de la Rubia and M.W. Guinan \\ Lawrence Livermore National Laboratory, L-644, Livermore, California 94550
}

(Received 14 May 1990; accepted 13 November 1990)

\begin{abstract}
We present results of a molecular dynamics simulation study of the effect of electron-ion interactions on the dynamics of the thermal spike in $\mathrm{Cu}$. Interatomic forces are described with a modified embedded atom method potential. We show that the electron-ion interaction acts to reduce the lifetime of the thermal spike and therefore the amount of atomic rearrangement that takes place in energetic displacement cascades in $\mathrm{Cu}$. The results point toward the important effect that inelastic energy losses might have on the dynamics of displacement cascades in the subcascade energy regime where the lifetime of the thermal spike is expected to exceed the electron-phonon coupling time.
\end{abstract}

\section{INTRODUCTION}

The characterization of the primary state of radiation damage in irradiated materials has been a problem of interest to the physics and materials research community for the last forty years. Component degradation under irradiation in fission and fusion reactors, ${ }^{1}$ as well as the use of modern ion beam techniques for materials modification, ${ }^{2}$ have motivated large research efforts aimed at achieving a basic understanding of this problem. Of fundamental importance in the characterization of the primary state of damage is understanding the role played by energetic displacement cascades. Displacement cascades, initiated when an energetic ion or neutron collides with an atom in the solid, deposit a large amount of energy in a localized region of the solid, thereby driving the system to a highly nonequilibrium state containing elevated defect concentrations and, in the case of irradiated alloys, disorder.

Due to their inhomogeneous and highly nonequilibrium nature, theoretical modeling of energetic displacement cascades has proven difficult. As a displacement cascade evolves and the characteristic energy of the atoms in the affected region falls below a few $\mathrm{eV}$, the theoretical description of the event must take into account the diffusive (as opposed to ballistic) nature of the process.

In the last few years, molecular dynamics computer simulation (MD) has been shown to be a useful tool to describe the dynamics and structure of energetic displacement cascades. For primary knock-on atom (PKA) energies up to several $\mathrm{keV}^{3,4}$ previous studies have shown that after a brief collisional phase lasting of the order of $10^{-13} \mathrm{~s}$, a cooling phase ensues in which atom trajectories are diffusive as opposed to ballistic. This phase, commonly termed a thermal spike, lasts several picoseconds and has been shown to be of critical importance in determining the amount of atomic rearrangement that takes place in energetic displacement cascades. In particular, the crucial role of cascade induced melting on total defect production, point defect clustering, and atomic mixing in $\mathrm{Cu}$ and $\mathrm{Ni}$ has been clearly demonstrated. ${ }^{4-6}$

Several problems concerning the proper physical description of a cascade event within an MD simulation remain. First, the interatomic potentials employed in previous studies considered only pair interactions, thus handicapping the correct description of the equilibrium properties of the metals under consideration. Furthermore, classical MD simulations describe the thermal behavior of an insulator. It thus becomes necessary to include in the simulations more realistic interatomic potentials and to account properly for inelastic energy loss $^{7}$ in the cascade as well as the thermal conductivity of the simulated crystal.

In this paper, we describe a model to account for both inelastic scattering and the thermal conductivity of the metal ${ }^{8}$ as well as the implementation of an isotropic many-body type potential based on the embedded atom method (EAM) of Daw and Baskes. ${ }^{9}$ EAM potentials have been recently used to identify the importance of many-body effects on atomic collisions in the low $\mathrm{eV}$ range ${ }^{10}$ and to study sputtering from metal surfaces. ${ }^{11}$

We identify two regimes of electron-ion interaction. ${ }^{8}$ The first, inelastic energy loss, is termed the electronic-stopping-power regime, and the second, the electron-phonon interaction regime. Together they cover a large energy range, extending from PKA to thermal energies. The model is then employed to study the thermal spike phase of cascades in $\mathrm{Cu}$ by consider- 
ing crystals in which the central region is heated to temperatures corresponding to atomic energy densities ranging from 5 to $10 \mathrm{eV} /$ atom.

In Sec. II we describe the input to the MD model employed in these simulations and give details of the MOLDYCASK code. ${ }^{12}$ Section III discusses the electronphonon interaction model and its implementation, paying particular attention to the determination of the electron-phonon coupling constants. Section IV describes modifications performed on the short-range part of the EAM potential employed for $\mathrm{Cu}$ in order to describe correctly the high energy scattering present in displacement cascade processes. In Sec. $\mathrm{V}$ we present the results of our MD simulations. The nonlinear nature of the electron-phonon coupling is clearly indicated. We show that the many-body potential for $\mathrm{Cu}$ results in reduced atomic motion in the cascade when compared to simulations with pair potentials. The inclusion of electronic losses correspondingly affects the lifetime as well as the amount of atomic mixing in the spike. We draw conclusions regarding the effect of the electron-phonon interaction in the dynamics of the spike and its possible influence on higher energy cascades in Sec. VI.

This work represents the first application of a realistic electron-ion interaction model to simulations of spike effects in $\mathrm{Cu}$. The introduction of this model and the application of a modified embedded atom method potential represent a substantial improvement over previous calculations in the description of the physical parameters relevant to simulations of the role of high energy displacement cascades in the formation of the primary state of damage in irradiated metals.

\section{MOLECULAR DYNAMICS MODEL}

The heat spike events described in the present paper have been carried out with a new MD code termed MOLDYCASK. This code is a modified version of the MOLDY6 program developed by Finnis ${ }^{13}$ and is described in detail in Ref. 12. In essence, the code employs the EAM potentials for describing the atomic interactions and implements a vectorized version of the link cell method (VLC) $)^{14,15}$ to update the neighbor tables. The VLC permits vectorization of the force calculation on a CRAY supercomputer, thereby greatly reducing the amount of CPU employed in the simulations. The crystallites employed were cubic cells containing 55296 atoms with periodic boundary conditions applied to all the faces of the MD cell. The simulations were carried out at constant volume. Damping was applied to the atoms in the boundary layers in order to describe correctly the dissipation of energy out of the crystal into an infinite continuum. The damping constant employed had a value of $1.4 \times 10^{-13} \mathrm{Kg} / \mathrm{s}$. The crystals were equilibrated at $10 \mathrm{~K}$ prior to the initiation of the spike event.
This was achieved by means of Langevin dynamics, which effectively couples the system to a thermal reservoir. $^{16}$ The equations of motion are integrated with a fourth order predictor-corrector algorithm with a variable time step. The code completes an MD iteration in $2.4 \times 10^{-4} \mathrm{~s}$ /atom of CPU on the CRAY-XMP computer at the Swiss Federal Institute of Technology in Zurich.

The simulations were performed by endowing the atoms in the center of the crystal with a Gaussian radial distribution of velocities corresponding to the desired energy in the spike. The width of the Gaussian was chosen as $1.5 a_{0}$, where $a_{0}$ is the lattice parameter of $\mathrm{Cu}$. Four events were simulated. These corresponded to total deposited energies of 2500 and $5000 \mathrm{eV}$, and to calculations with and without the inclusion of electronic losses. The energy densities in the spike region were 5 and $10 \mathrm{eV} /$ atom, respectively.

\section{ELECTRONIC LOSSES MODEL}

In a recent paper $^{8}$ we have shown how, using the local density formulation of the stopping power, ${ }^{17}$ the electronic losses of high energy particles can be incorporated into an MD simulation using the Langevin equations of motion. The same formalism describes the electron-phonon interaction, and using the local density formalism both regimes are merged. In this model, the damping term in the Langevin equation, which is responsible for the decay of energy fluctuations in the system, becomes a function of the local electronic density. The term representing the energy exchange is a stochastic force acting on the ions and depends on the electronic temperature and the damping coefficient. The action of both terms brings the system into thermal equilibrium.

A model of the ion-electron coupling should also include the evolution of the electronic temperature. However, the problem is far too complex to be fully described in the context of an MD simulation of a crystal containing several tens of thousands of atoms. Nevertheless, a simple estimate of the influence of the electronic heating may be made in two limiting cases. First, we assume the electron and phonon systems to be weakly coupled; then when energy is deposited into the ion system, the ions will reach equilibrium through the phonon-phonon interaction and only a small amount of energy will be transferred to the electrons. Since the coupling is small, the electronic mean free path will be large compared to the cascade dimensions (typically of the order of $30 \AA$ in radius). Therefore, the excited electrons will decay far from the cascade region, producing no significant increase in the electronic temperature. In other words, for a weakly coupled system such as a noble metal, a reasonable approximation is to assume that the electronic temperature remains constant throughout the simulation. The other limiting case is 
that of strongly coupled systems, such as transition metals. Here, the mean free path of the excited electrons might be small compared to the cascade dimensions and the electrons might decay inside the cascade core. These two limiting cases can easily be modeled in a computer simulation; nevertheless, the situation in actual materials is expected to lie somewhere in between. It is interesting to note in this context that Flynn and Averback ${ }^{18}$ have shown that as a thermal spike develops, a given system may oscillate between the two limiting situations; weak to strong coupling and then weak coupling again. The aim of the present paper is to apply the ion-electron interaction model to a weakly coupled system such as copper, where it is assumed that the electronic temperature remains constant throughout the simulation. In the following, we describe the implementation of this model in our MD simulations.

\section{Implementation of the electronic loss model in the MD simulations}

A correction to the calculated forces has been introduced in MOLDYCASK according to the Langevin equation of motion which reads

$$
m \cdot \ddot{x}_{i}=F_{i}+\eta_{i}(t)+\beta_{i} \cdot \dot{x}_{i}
$$

where $\eta_{i}$ is a random force defined by:

$$
\begin{aligned}
\left\langle\eta_{i}(t)\right\rangle & =0 \\
\left\langle\eta_{i}(t) \cdot \eta_{i}\left(t^{\prime}\right)\right\rangle & =2 \cdot \pi \cdot K_{B} \cdot T \cdot \delta\left(t-t^{\prime}\right)
\end{aligned}
$$

and with the distribution

$$
P\left(\eta_{i}\right)=\left(2 \pi\left\langle\eta^{2}\right\rangle\right)^{-1 / 2} \cdot \exp \left(-\frac{\eta^{2}}{2\left\langle\eta^{2}\right\rangle}\right)
$$

In this expression $T$ is the constant temperature of the electron bath, which has been chosen equal to the initial temperature of the equilibrated crystal. The damping term $\beta_{i}$ has been described according to the following expression, which is a modification of that given in Ref. 8:

$$
\beta_{i}=A \cdot Z^{2} \cdot \log \left(B \cdot \rho_{i}^{1 / 3}+C\right)+b_{i}
$$

As mentioned above, the boundary damping constant $b_{i}$ has been evaluated as $0.14 \times 10^{-12} \mathrm{~kg} / \mathrm{s}$ for atoms within the boundary layers and 0.0 otherwise.

From Ref. $8, A=2 e^{4} m^{2} / 3 \pi \hbar^{3}=7.983 \times$ $10^{-15} \mathrm{Js} / \mathrm{m}^{2}$ and $B=\left(3 \pi^{2}\right)^{1 / 2} \hbar^{2} / e^{2} m=1.63710 \times$ $10^{-10} \mathrm{~m}$. The model has two adjustable parameters, namely $Z$ and $C$, that must be chosen to reproduce the high energy stopping power (depending essentially on $Z$ ) and the electron-phonon coupling at room temperature (depending both on $Z$ and $C$ ). To proceed with the evaluation of these two parameters, a simple method has been used based on heating, by means of the electrons, a system initially in thermal equilibrium. In this manner, an average coupling time constant may be obtained. In order to obtain an accurate evaluation of this time scale, we chose to represent the strength of the electron-phonon coupling in terms of the dimensionless parameter $\lambda$ entering the McMillan theory of superconductivity. ${ }^{19}$ Estimates of the magnitude of $\lambda$ may be obtained following two different approaches. The first is based on time-resolved experimental observations of the thermal relaxation of hot electrons in metals. ${ }^{20}$ In these experiments, a very short laser pulse directed at the surface of a metal creates a nonequilibrium electron distribution while leaving the lattice temperature unchanged. Over a time scale of a few picoseconds the electrons are observed to return to local equilibrium at a somewhat elevated temperature. These experiments, when interpreted in terms of the theory of electronic relaxation proposed by Allen, ${ }^{21}$ allow a determination of the parameter $\lambda$ for $\mathrm{Cu}$ between 0.1 and 0.2 .

A second determination of $\lambda$ comes from band theory values for the density of states at the Fermi level and the Drude plasma frequency. This estimation of $\lambda$ results in a value for $\mathrm{Cu}$ of $0.13 .^{22}$

The scattering rate of electrons is obtained from $\lambda$ through:

$$
\frac{1}{\tau_{e-p h}}=\frac{2 \cdot \pi \cdot \lambda \cdot k_{B} \cdot T}{h}
$$

The lifetimes of phonons and electrons are different because the numbers of them available to undergo collisions are different. Nevertheless, the lifetime of the fluctuations of energy decaying into electrons is the same, because of energy conservation, to the lifetime of the fluctuations decaying into the ions. The phonon lifetime is given by:

$$
\tau_{p h-e}=\tau_{e-p h} \cdot \frac{T_{\mathrm{F}}}{\theta_{\mathrm{D}}}
$$

where $T_{\mathrm{F}}$ and $\theta_{\mathrm{D}}$ are the Fermi and the Debye temperatures, respectively. Using values of $T_{\mathrm{F}}=8.16 \times 10^{4} \mathrm{~K}$ and $\theta_{\mathrm{D}}=315 \mathrm{~K},{ }^{23}$ coupling constants of 3.4 and $7.0 \mathrm{ps}$ are obtained for $\lambda=0.2$ and 0.1 , respectively.

In the simulations, we employ an average value of the coupling constant in the Langevin equations of 6 ps and obtain a value for the effective charge $Z=3.0$ and $C=0.84$.

\section{MODIFIED EAM POTENTIALS}

We have employed an interatomic potential based on the embedded atom method of Daw and Baskes ${ }^{9}$ to simulate $\mathrm{Cu}$. Following the method of Foiles, Baskes, and Daw ${ }^{24}$ the potential was fitted to equilibrium data of $\mathrm{Cu}$, and to data on the dilute limits of the heats of solution of alloys of six transition metals, namely $\mathrm{Ni}$, $\mathrm{Pd}, \mathrm{Pt}, \mathrm{Cu}, \mathrm{Ag}$, and $\mathrm{Au}$. Since the fit makes use of the zero temperature and pressure equation of state of Rose 
et al., ${ }^{25}$ the elastic constants, bulk modulus, and equilibrium lattice parameter of copper were extrapolated to $0 \mathrm{~K}$. The data employed, as well as a complete description of the fitting procedure, are given in Ref. 12 .

In order to describe correctly the elastic scattering at the high particle energies present in an energetic displacement cascade, the high energy part of the potential has been further modified. At short range, one expects the scattering to be dominated by the repulsive wall of the potential, thus making a pair potential description appropriate. To this end, we have modified the embedding function as well as the pair potential contribution to the total energy of $\mathrm{Cu}$ for interaction distances smaller than those typical of threshold displacement events. This is justified in view of the correct description of the threshold energy of $\mathrm{Cu}$ obtained with the unmodified EAM potential. ${ }^{26}$ In order to describe properly the high-energy electronic energy loss, the atomic density functions of the EAM have been modified so as to reproduce the full Thomas-Fermi electron density at short range. Details of the modifications performed are presented in the appendix.

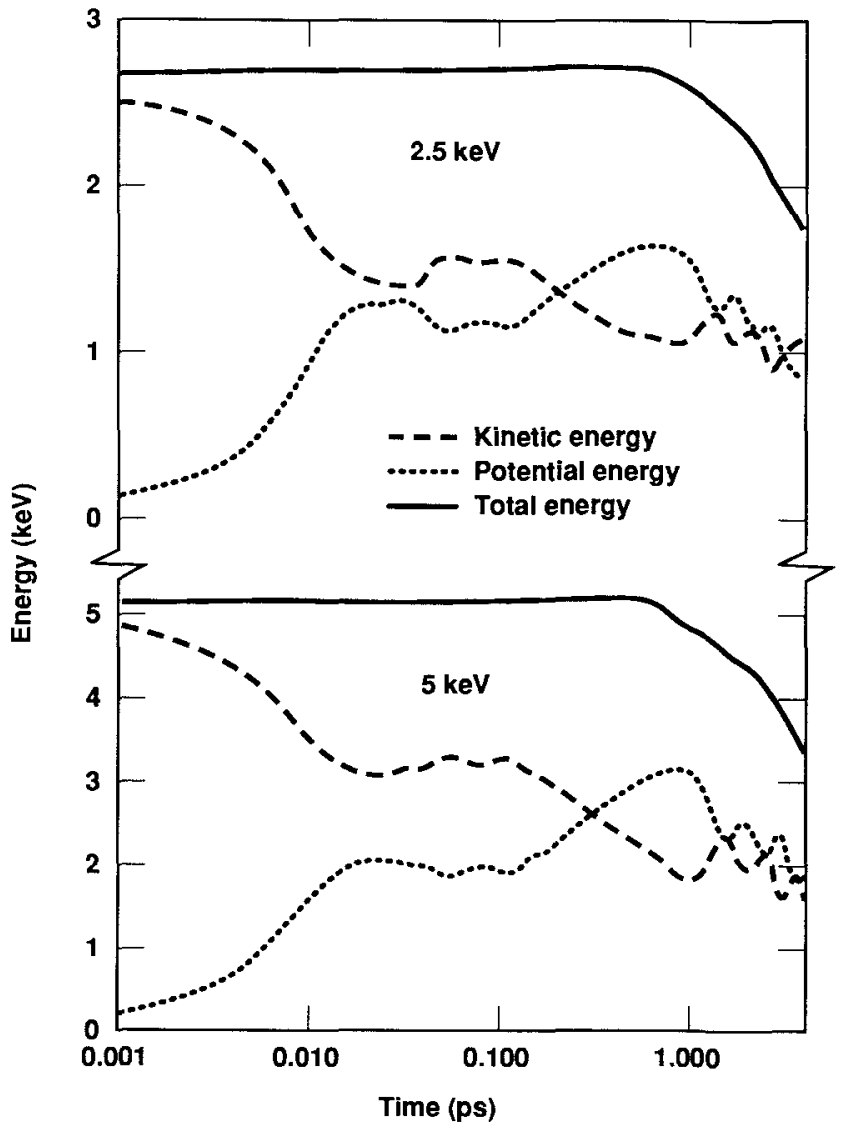

(a)

\section{RESULTS}

In order to evaluate the effect of the electronic losses and to separate this effect from the artificial energy damping imposed at the boundaries, two runs at each of the two energies considered have been done: one with electronic losses and one without them, the latter providing the reference frame. Figures $1(a)$ and 1(b) show the two runs at each energy, 2.5 and $5 \mathrm{keV}$. The curves represent the kinetic, potential, and total energies in the crystal, measured as the difference from the values in static equilibrium. Energy is observed to reach the boundaries at about $0.8 \mathrm{ps}$ in all runs.

To investigate the influence of the electronic system on the total energy, we define $E_{e}(t)$ and $E_{0}(t)$ as the total crystal energy for the cases with and without electronic losses included in the simulation. To avoid including the effect of the artificial damping imposed at the boundaries in the analysis, we define the quantity $\left(Y(t)=\log \left[E_{e}(t) / E_{0}(t)\right]\right.$. If the coupling between the electrons and the lattice were constant throughout the spike lifetime $Y(t)$ should be a linear function of time

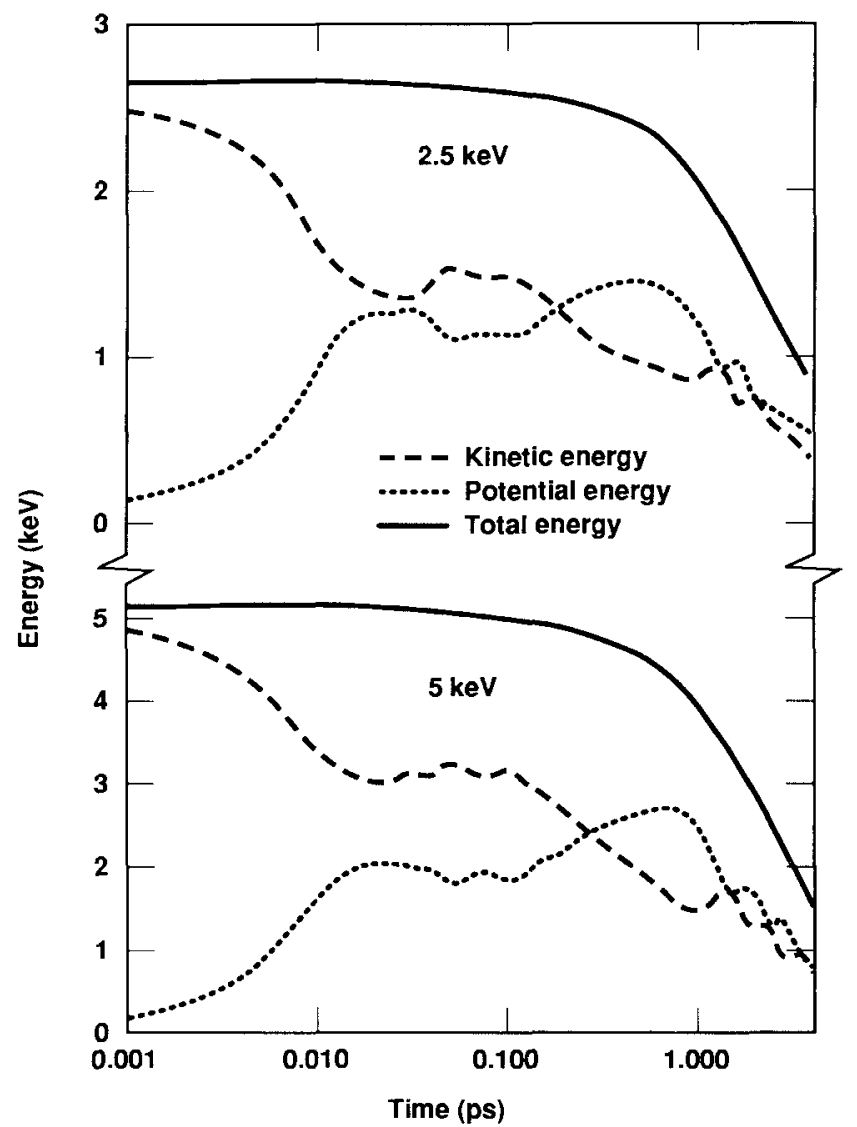

(b)

FIG. 1. Time evolution of the total, kinetic, and potential energy of the computational crystallites for the four events studied. (a) 2.5 and $5 \mathrm{keV}$ heat spikes without electronic coupling; (b) 2.5 and $5 \mathrm{keV}$ heat spikes with electronic coupling. Energies are measured with respect to the static equilibrium crystal configuration. 
with slope $-1 / \tau$. In order to obtain the time dependence the coupling constant, we define $W(t)=$ $-1 /[d Y(t) / d t]=\tau$. In Fig. 2 we plot $Y(t)$ versus time for the 2.5 and $5.0 \mathrm{keV}$ runs. Several important conclusions may be reached from this figure. The figure clearly shows that the total energy does not decrease linearly in the logarithmic scale. This effect reproduces the temperature dependence of the electron-phonon coupling. On the right axis of Fig. 2, we plot the value of $W(t)$, i.e., the inverse of the coupling, or the lifetime of the fluctuations. While it has been chosen as 6 ps at low temperature, it is seen from the figure that $\tau$ ranges in value from 2 to $6 \mathrm{ps}$. At the early stage of the heat spike the coupling increases by a factor of two, giving an instantaneous lifetime of the fluctuations of about $3 \mathrm{ps.}$

The disordering tendency of the $5000 \mathrm{eV}$ spike event in $\mathrm{Cu}$ is demonstrated in Figs. 3(a)-3(d), where instantaneous atomic configurations in a (010) plane through the center of the spike are shown at two different times. As can be seen from Figs. 3(a) and 3(b), the atomic rearrangement lasts of the order of $4.5 \mathrm{ps}$ in the

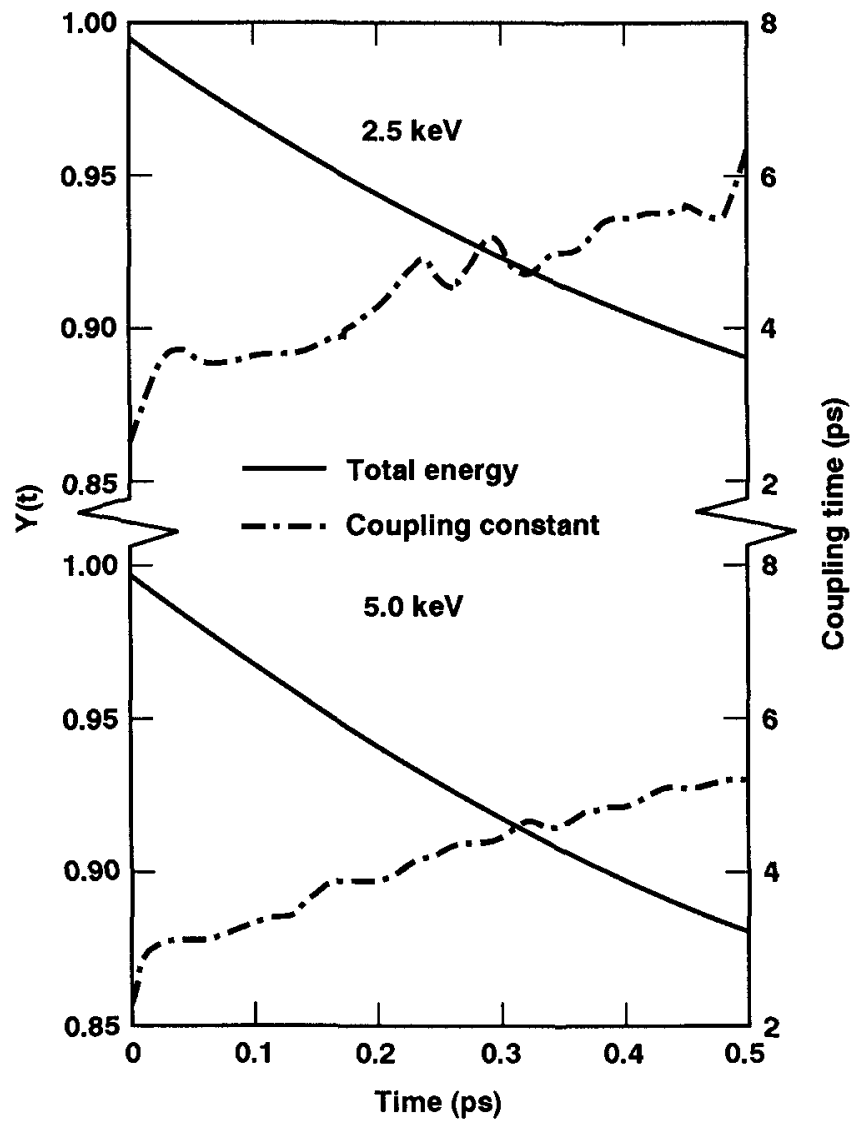

FIG. 2. Logarithm of the ratio of the total energy of the crystal for the run with electronic losses to that of the reference run versus time for the two spike energies considered $[Y(t)$ in the text]. On the right ordinate, we plot the inverse of the logarithmic derivative of the ratio of the total energies, which is equal to the electron phonon coupling constant $\tau$. case where electronic losses are included and more than 5 ps without them. A similar effect is observed for the $2500 \mathrm{eV}$ events. To quantify further the dynamics of the cascade, we have evaluated the temperature as a function of distance from the center of the spike region, and from this we have extracted information regarding the radius of the region that remains at a temperature above the melting point of $\mathrm{Cu}, R_{m}(t)$. This is shown in Fig. 4. While the melted zone has already disappeared in the case where electronic losses are present by about $3 \mathrm{ps}$, there is still a melted region of about $12 \AA$ in radius in the case without losses.

Heat spike simulations also provide information on the magnitude of atomic mixing in the cascade. Following Refs. 4 and 5, we define atomic mixing by the mean square displacement per atom in the cascade

$$
\left\langle\delta r^{2}(t)\right\rangle=\frac{1}{N} \cdot \sum_{i-1}^{N}\left[r_{i}(t)-r_{i}(0)\right]^{2}
$$

where $r_{i}(0)$ denotes the perfect lattice position of atom $i$, whereas $r_{i}(t)$ indicates its displaced position at time $t$. The evolution of this measure is shown in Fig. 5. The maximum observed at 0.8 ps can be associated with the high energy density introduced by the heat spike into an otherwise equilibrium crystal. By the end of the events, a small difference between the cases with and without electronic losses of about $20 \%$ can be seen for the $5000 \mathrm{eV}$ events and about $10 \%$ for the $2500 \mathrm{eV}$ events. The weakness of this final effect was expected in $\mathrm{Cu}$ since the characteristic coupling time of $6 \mathrm{ps}$ for the ion-electron interaction is comparable to the lifetime of the spikes. In order to compare quantitatively the simulation results with experimental values, the values of the total mean square displacement obtained in the present simulations, $\Sigma r_{i}{ }^{2}$, have been normalized by the damage energy. Thus, we define the mixing efficiency as

$$
\frac{D t}{\phi F_{D}}=\frac{1}{6} \cdot \sum_{i} \frac{\vec{r}_{i}^{2}}{n_{0} E_{D}^{\prime}}
$$

where $\phi$ is the ion dose in an experiment, $F_{D}$ is the damage energy per unit volume deposited perpendicular to the surface, $n_{0}$ is the atomic density of the target, and $E^{\prime}{ }_{D}$ is the damage energy per atom in the simulation. In Table I we present values of the mixing efficiency obtained from these and previous ${ }^{5}$ heat-spike simulations as well as experimental results for the mixing of thin markers in $\mathrm{Cu}^{27}$ Note that although the magnitude of atomic mixing is comparable in all these events, the previously reported results, corresponding to calculations with pair potentials, corresponded to much lower energy density heat spikes. While 1.1 and $2.8 \mathrm{eV} /$ atom heat spikes produce values of atomic mixing of 12 and $22 \mathrm{~A}^{5} / \mathrm{eV}$, respectively, for the case of pair interactions, a $5 \mathrm{eV} /$ atom spike in $\mathrm{Cu}$ with the EAM 


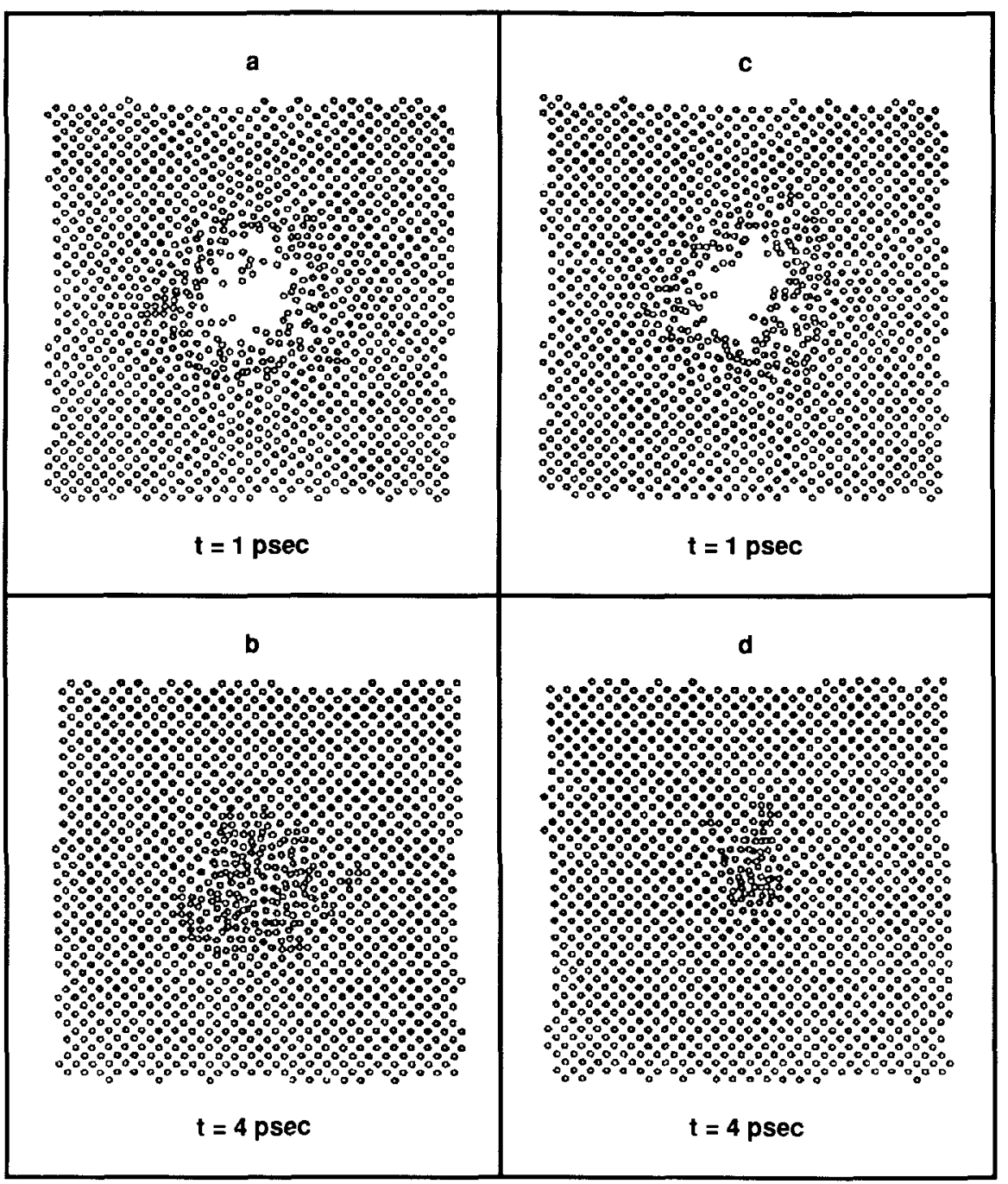

FIG. 3. Cross-sectional views of the $5 \mathrm{keV}$ heat spikes at two different times. The plots represent cuts through a (100) plane. $(a, b)$ No electronic coupling, at $t=1$ and $t=4$ ps. (c, d) With electronic coupling at $t=1$ and $t=4 \mathrm{ps}$.

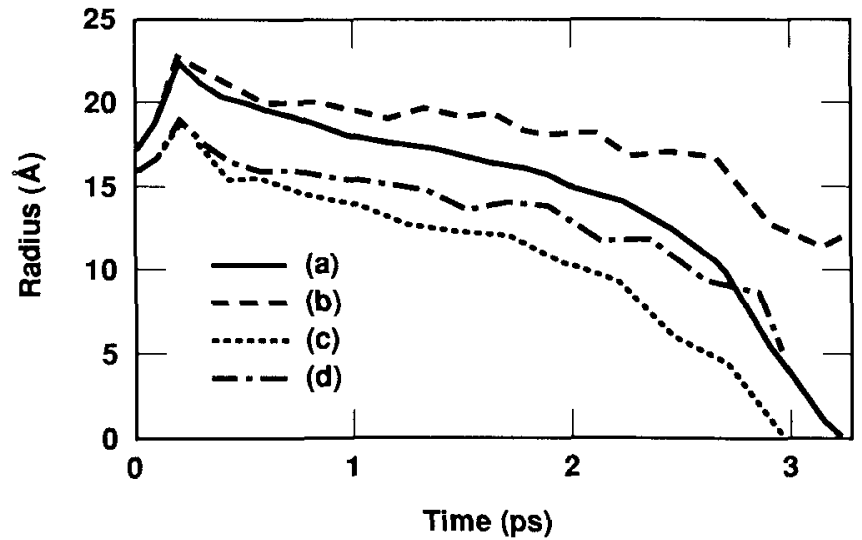

FIG. 4. Radius of the regions where the temperature in the spike is above the experimental melting temperature of $\mathrm{Cu}$ for the four events considered. (a, b) $5 \mathrm{keV}$ events with and without the electronic coupling, respectively. (c, d) $2.5 \mathrm{keV}$ events, same as before. potential produces a value of mixing of only $24 \mathrm{~A}^{5} / \mathrm{eV}$. This effect is clearly due to the difference in the description of the properties of $\mathrm{Cu}$ with the two potentials mentioned and not to the inclusion in the model of inelastic energy losses. While the EAM potential gives results for the point defect formation and migration energies, as well as formation volumes in quite good agreement with experiment, ${ }^{12}$ the Born-Mayer pair potential employed in previous simulations is known to underestimate the self interstitial (SIA) and vacancy migration energies by about a factor of two and the divacancy migration energy by over a factor of 10 , resulting in divacancy migration in stage $\mathrm{I}^{28}$

\section{CONCLUSIONS}

The important role of cascade-induced melting on total defect production, defect clustering, and atomic 


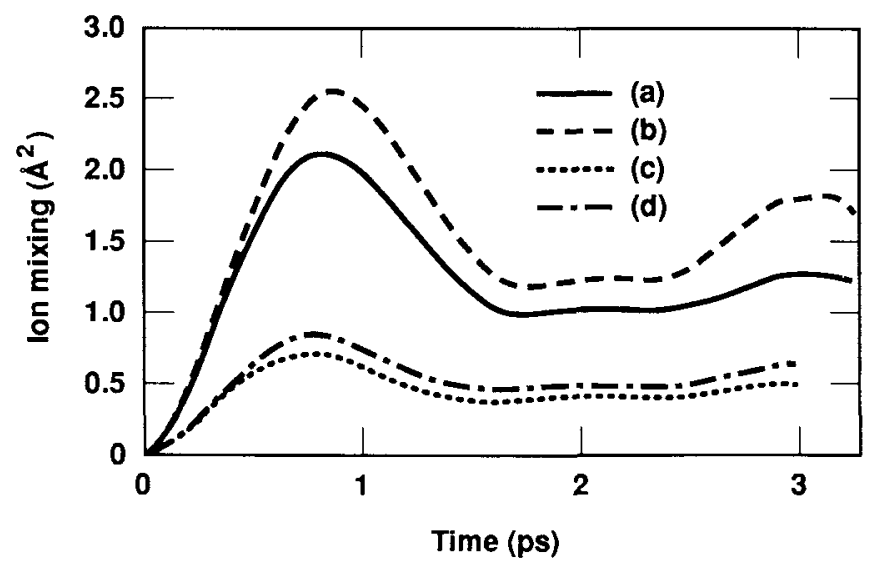

FIG. 5. Ion mixing as defined in Eq. (7). (a, b) $5 \mathrm{keV}$ events with and without the electronic coupling, respectively. (c, d) $2.5 \mathrm{keV}$ events, same as before.

mixing in cascades has been clearly demonstrated in the literature. ${ }^{4,5}$ The present study of heat spikes in $\mathrm{Cu}$, although resulting in somewhat different quantitative results attributed to the use of a many-body potential, has shown similar qualitative features of the structure and dynamics of spikes in $\mathrm{Cu}$ as those previously reported. More importantly, the present simulation has shown the important effect that inelastic energy losses might have in the evolution of energetic displacement cascades. We have observed a moderate decrease of the disordered zone lifetime when electronic losses are included with a concurrent decrease in atomic mixing.

These two effects are present in $\mathrm{Cu}$, the most unfavorable example, as the ion-electron coupling is weak in this case. In other materials such as $\mathrm{Ni}$, where the ion-electron interaction is expected to be stronger, the difference is expected to become important already for events in the low-keV range of energies. In this case, however, the approximation of a fixed electron bath temperature is no longer valid and a model for the electron heat transport must be included in the calculation.

TABLE I. Mixing efficiency for $\mathrm{Cu}$. Results include previous simulation studies with pair potentials and experimental results.

\begin{tabular}{cc}
\hline \hline Energy density (eV/atom) & $D t / \phi F_{D}\left(\AA^{5} / \mathrm{eV}\right)$ \\
\hline $1.1^{\mathrm{a}}$ & 12 \\
$1.9^{\mathrm{a}}$ & 18 \\
$2.8^{\mathrm{a}}$ & 22 \\
$5^{\mathrm{b}}$ & 26 \\
$5^{\mathrm{c}}$ & 24 \\
$10^{\mathrm{b}}$ & 38 \\
$10^{\mathrm{c}}$ & 32 \\
Experiment $^{\mathrm{d}}$ & 25 \\
\hline
\end{tabular}

${ }^{\mathrm{a}}$ Ref. 5.

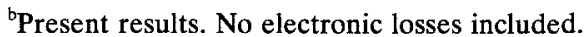

${ }^{\mathrm{c}}$ Present results. Electronic losses included.

${ }^{\mathrm{d}}$ Ref. 26.
We conclude by pointing out that for higher energy events the lifetime of the cascade will increase, ${ }^{29}$ up to the subcascade breaking point, and therefore a significant effect may be expected even in the case of weakly coupled copper.

\section{ACKNOWLEDGMENTS}

One of the authors (TDR) wishes to acknowledge the hospitality of the Paul Scherrer Institute while on a visit during which part of this work was completed. This work has been partially supported by the National Energie-Forschung-Fonfs, under Contract No. 332, the Fonds National Suisse de la Recherche Scientifique, Grant No. 2.866-0.88, and was carried out under the auspices of the United States Department of Energy by Lawrence Livermore National Laboratory under Contract W-7405-Eng-48.

\section{REFERENCES}

${ }^{1}$ Proceedings of Workshop on Fusion Materials, Lugano, Switzerland, May 1988, Rad. Eff. Def. Sol. 113 (1990).

${ }^{2}$ See, e.g., Beam-Solid Interactions and Transient Processes, edited by M. O. Thompson, S.T. Picraux, and J. S. Williams (Mater. Res. Soc. Symp. Proc. 74, Pittsburgh, PA, 1987).

${ }^{3}$ M.W. Guinan and J. Kinney, J. Nucl. Mater. 103/104, 1319 (1981).

${ }^{4}$ T. Diaz de la Rubia, R. S. Averback, R. Benedek, and W. E. King, Phys. Rev. Lett. 59, 1930 (1987).

${ }^{5}$ T. Diaz de la Rubia, R. S. Averback, H. Hsieh, and R. Benedek, J. Mater. Res. 4, 579 (1989).

${ }^{6}$ H. Hsieh, T. Diaz de la Rubia, R. S. Averback, and R. Benedek, Phys. Rev. B 40, 9986 (1989).

${ }^{7}$ J. Lindhard, M. Scharff, and H. E. Schiøtt, Mat.-Fys. Medd. K. Dan. Vidensk. Selsk. 33, No. 14 (1963).

${ }^{8}$ A. Caro and M. Victoria, Phys. Rev. A 40, 2287 (1989).

${ }^{9}$ M. Daw and M. Baskes, Phys. Rev. B 29, 6443 (1984).

${ }^{10}$ S. P. Chou and N. M. Ghoniem, presented at the IV ICFRM, Kyoto, Japan, 1989; to be published in J. Nucl. Mater.

${ }^{11}$ B. J. Garrison, N. Winograd, D. M. Deaven, C.T. Reimann, D.Y. Lo, T. A. Tombrello, D.E. Harrison, Jr., and M. H. Shapiro, Phys. Rev. B 37, 7197 (1988).

${ }^{12}$ T. Diaz de la Rubia and M.W. Guinan, Proceedings of Workshop on Fusion Materials, Silkeborg, Denmark, 1989 (to be published in J. Nucl. Mater.) and University of California Report UCRL103511 (1990).

${ }^{13}$ M. Finnis, "MOLDY6-A Molecular Dynamics Program for Simulation of Pure Metals", Harwell Rept. AERE-R-13182 (1989).

${ }^{14}$ D. Fincham and D. M. Heyes, in Advances in Chemical Physics, edited by M.W. Evans (Wiley, New York, 1985), Vol. LXIII, p. 493.

${ }^{15}$ D. M. Heyes and W. Smith, in Information Quarterly for Computer Simulation of Condensed Phases, No. 26 (Science and Engineering Research Council, Daresbury Laboratory, Daresbury, England, 1987), p. 68.

${ }^{16}$ See, e.g., R. Biswas and D. R. Hamann, Phys. Rev. B 34, 895 (1986) and references therein.

${ }^{17}$ P. M. Echenique, R. M. Nieminen, and R. H. Ritchie, Solid State Commun. 37, 779 (1981).

${ }^{18}$ C. P. Flynn and R. S. Averback, Phys. Rev. B 38, 7118 (1988).

${ }^{19}$ W. I. McMillan, Phys. Rev. 167, 331 (1968).

${ }^{20}$ H. E. Elsayed-Ali, T. B. Norris, M. A. Pressot, and G. A. Mourou, Phys. Rev. Lett. 58, 1212 (1987). 
${ }^{21}$ P. B. Allen, Phys. Rev, Lett. 59, 1460 (1987).

${ }^{22}$ P. B. Allen, Phys. Rev. B 36, 2920 (1987).

${ }^{23} \mathrm{C}$. Kittel, Introduction to Solid State Physics, 2nd ed. (Wiley, New York, 1963).

${ }^{24}$ S. Foiles, M. Baskes, and M. S. Daw, Phys. Rev, B 33, 7983 (1986).

${ }^{25}$ J. H. Rose, J. R. Smith, F. Guinea, and J. Ferrante, Phys. Rev. B 29, 2963 (1984).

${ }^{26}$ T. Diaz de la Rubia, Ph. D. Thesis, State University of New York at Albany, Albany, NY (1989).

${ }^{27}$ S-J. Kim, M-A. Nicolet, R. S. Averback, and D. Peak, Phys. Rev. B 37, 38 (1988).

${ }^{28}$ P. H. Dederichs, C. Lehmann, H. R. Schober, A. Scholz, and R. Zeller, J. Nucl. Mater. 69/70, 176 (1978).

${ }^{29}$ P. Sigmund, Appl. Phys. Lett. 25, 169 (1974).

${ }^{30}$ W. D. Wilson, L. G. Haggmark, and J. P. Biersack, Phys. Rev. B 15, 2458 (1977).

${ }^{31}$ J. Ziegler, J.P. Biersack, and U. Littmark, The Stopping and Range of Ions in Solids (Pergamon, New York, 1985), Vol. 1.

\section{APPENDIX}

Following Foiles et al. ${ }^{24}$ the total energy of an fcc metal in the EAM is given as

$$
E_{\mathrm{tot}}=\sum_{i} F_{i}\left(\rho_{h, i}\right)+\frac{1}{2} \sum_{i \neq j} \phi_{i, j}\left(r_{i, j}\right)
$$

where $F$, the embedding term, is a functional of the background electron density experienced by atom $i$, and $\phi$ is a pair potential term. The background electron density is expressed as a superposition of atomic $s$ and $d$ valence electron densities with no core electrons entering the expression for $\rho$. The total energy is expressed in terms of the equation of state of Rose et al. ${ }^{25}$

$$
E_{\mathrm{tot}}=-E_{\mathrm{coh}} \cdot\left(1+a^{*}+b \cdot a^{* 3}+\cdots\right) \cdot \exp \left(-a^{*}\right)
$$

where the $a^{* 3}$ term was included in Ref. 12 in order to obtain a better description of the pressure derivative of the isothermal bulk modulus of $\mathrm{Cu} . E_{\text {coh }}$ is the equilibrium cohesive energy per atom, and

$$
a^{*}=\left(\frac{a}{a_{0}}-1\right) /\left(E_{\mathrm{coh}} / 9 \cdot B \cdot \Omega\right)^{1 / 2}
$$

and $a_{0}, B$, and $\Omega$ are the equilibrium lattice parameter, isothermal bulk modulus, and atomic volume, respectively.

As mentioned in the text, when dealing with the energies characteristic of recoil atoms in displacement cascades, it becomes necessary to modify the shortrange part of the EAM potential. To this end, we have modified the embedding function and pair potential terms in Eq. (A1) above. So as to describe properly the high energy part of the inelastic stopping power within the EAM model, we have also modified the form of the atomic electron density for interaction distances less than $1 \AA$. Note that in our model, the electron-ion interaction enters via the electron density dependent damping term in the Langevin equation of motion.

\section{A. Modification of the embedding function}

The form of the embedding function, $F$, used in this work is

$$
\begin{gathered}
F=F^{\operatorname{EAM}}(\rho) \\
\rho<0.1 e^{-} / \AA^{3} \\
F=A_{0}+A_{1} \rho+A_{2} \rho^{2}+A_{3} \rho^{3}+A_{4} \rho^{4} \\
0.1 \leqslant \rho<0.136 e^{-} / \AA^{3} \\
F=\text { constant }=-10.84 \mathrm{eV} \begin{array}{l}
\rho \geqslant 0.136 e^{-} / \AA^{3}
\end{array}
\end{gathered}
$$

where the polynomial coefficients are given in Table AI.

\section{B. Modification of the pair potential}

The pair potential has been modified so as to match the form of the $\mathrm{Kr}-\mathrm{C}$ universal potential. ${ }^{30}$ This is a screened-Coulomb potential which gives a good representation of high energy scattering of atoms in solids. The resulting pair potential function is given as

$$
\begin{gathered}
\phi=\frac{Z^{2}}{r} \cdot\left[0.191 e^{-2.9054 r}+0.474 e^{-6.633 r}\right. \\
\left.+0.335 e^{-19.984}\right] \\
r \leqslant 0.8 \AA \\
\phi=\exp \left(A_{0}+A_{1} r+A_{2} r^{2}+A_{3} r^{3}\right) \\
0.8<r \leqslant 1.3 \AA \\
\phi=\phi^{\mathrm{EAM}}=\frac{Z_{\text {eff }}^{2}}{r} \\
r>1.3 \AA
\end{gathered}
$$

TABLE AI. Coefficients of the fitting polynomials for the embedding function, pair potential, and atomic electron density employed in the modified EAM potential.

\begin{tabular}{rrrrrr}
\hline & \multicolumn{1}{c}{$A_{0}$} & \multicolumn{1}{c}{$A_{1}$} & \multicolumn{1}{c}{$A_{2}$} & \multicolumn{1}{c}{$A_{3}$} & $A_{4}$ \\
\hline$F$ & -92.48 & 3112.75 & -41752.03 & 238926.43 & \\
$\phi$ & 7.01 & 4.06 & -9.77 & 3.41 & 1185.54 \\
$\rho$ & 189.84 & -1010.59 & 2147.65 & 2268.29 & -245.54 \\
\hline \hline
\end{tabular}


where $Z_{\text {eff }}$ for the EAM part of the potential is given as

$$
\mathrm{Z}_{\text {eff }}=Z_{0} \cdot\left(1+\beta r^{2}\right) \cdot e^{-\alpha r}
$$

and $Z_{0}$ is the number of valence electrons of $\mathrm{Cu}$, and $\beta$ and $\alpha$ have values of 0.02455 and 1.5362 , respectively. ${ }^{12}$ The values of the coefficients of the interpolation polynomial are given in Table AI. With this interpolation scheme, the value and the first derivative of the pair potential are guaranteed to be correct at the interpolation points.

\section{Atomic electron density}

The atomic charge density was modified at short range by means of an interpolation function joining the valence charge used in the EAM to the Thomas-Fermi form of the atomic electron density of $\mathrm{Cu}^{31}$ The final form of the charge density is

$$
\begin{array}{cc}
\rho=\rho^{\mathrm{T}-\mathrm{F}} & r \leqslant 0.7 \AA \\
\rho=\operatorname{Exp}\left(A_{0}+A_{1} r+A_{2} r^{2}+A_{3} r^{3}+A_{4} r^{4}\right. \\
\left.+A_{5} r^{5}\right) \\
\rho=\rho^{\mathrm{EAM}} \\
0.7<r \leqslant 1.0 \AA \\
\\
\end{array}
$$

